\title{
A Survey on Disaster Management Systems
}

\author{
Anjana Sara Thomas \\ Dept. of Computer Science \& Engineering \\ Mar Baselios College of Engineering \& Technology \\ Trivandrum, India
}

\author{
R. Vikraman Nair, PhD \\ Dept. of Computer Science \& Engineering \\ Mar Baselios College of Engineering \& Technology \\ Trivandrum, India
}

\begin{abstract}
Disaster is a sudden event bringing great damage to life and property. Managing a disaster situation is a challenging task. Disaster management is one of the most demanding applications in the field of emergency assistance and long term maintenance for refugees and displaced persons. One tool for improving disaster is quick access to accurate and updated information about the disaster situation. This paper aims to discuss various disaster management techniques that can be used by the first responders and the public to lessen the impacts caused during a disaster. The various disaster management systems reviewed in this paper includes $\mathrm{TtT}$ system, MRCCFR system, UbAlert disaster application and BRIDGE system. The advantages and disadvantages of using each of these systems are also discussed.
\end{abstract}

\section{General Terms}

Disaster, Disaster Management.

\section{Keywords}

Tweek-the-Tweet (TtT) System; Mobile Rich Media Communication Tool for First Responders (MRCCFR); Intelligent Guards Against Disasters (iGaDs); Bridging Resources and Agenices in Large Scale Emergency Management (BRIDGE).

\section{INTRODUCTION}

An emergency is a situation that causes an immediate risk to human health, life and property. Disasters can be natural or man-made. In case of a disaster, a co-ordinated effort by government and non government agencies are required to provide a relief from the impact of the disaster. Thus an emergency situation requires an urgent intervention. These interventions are usually organized as a process named as an emergency response process and are described in an emergency plan. Emergency management involves several activities. These include training personnel and developing tools and strategies used by the emergency organizations for responding and recovering from disasters. Accessing information regarding the disaster situation is of vital importance for emergency management to enable distribution of the right resources to the right group of people. Disasters occur in varied forms. Some are predictable in advance. Some are annual or seasonal and some are sudden and unpredictable.

The emergency situation can be handled effectively through the use of a wide variety of technologies. Disaster management systems can be used to help crisis managers and members of the public for hazard detection, prediction and for devising risk minimizing evacuation plans.Technology assisted disaster management methods offer a number of unique advantages over the approaches of disaster management systems that lack technological support [1]. User training is essential for disaster management concepts to display new technologies as tools for supporting disaster management. After recent Uttarakhand natural disaster, the Haryana government is planned to train 4000 disaster management personal immediately [2]. Virtual worlds or multi-user virtual environments have become a choice for technology enhanced learning [14]. Working through the disaster management system is very large and comprehensive. Disaster management doesn't eliminate the threats, instead it decreases the impact of disasters. Failure to create an efficient recovery plan results in serious damage to life and property.

In section II a brief description of the background is given. The various disaster management systems are discussed in section III.

\section{BACKGROUND}

Disaster is an event which is generally unpredictable. It happens instantly and affects a large number of people. The five phases of disaster management includes disaster preparedness, disaster impact, disaster response, rehabilitation and disaster mitigation.

Disaster preparedness aims at minimizing the adverse effects of a hazard. These activities provide permanent protection from disasters. Most disasters have sudden impact whereas some are 'slow onset' disasters. Natural disasters can be prevented through disaster prevention. In January 2005, 168 governments adopted a 10 year global plan for natural disaster reduction called the Hyogo framework. This framework offers guiding principles and practical means to lessen the impact of the disaster [3]. Preparedness can be in varied forms including money, manpower and materials. Disaster preparedness includes several activities including evaluation from past experiences about the risk, location of disaster prone areas, organization of communication information and warning system, ensuring co-ordination and response mechanisms, development of public education programme, co-ordination with media, national and international relations, keeping stock of other essential commodities. Disaster impacts can be classified as physical and social impact. The physical impacts can be easily measured and are first reported by the news media. Social impacts include psychosocial, demographic, economic and political impacts. Social impacts are difficult to assess when they occur. Disaster response provides immediate assistance to maintain life and to improve health of the affected population. Such assistance includes activities like giving warning, search and rescue, providing immediate assistance, assessing damage, continuing assistance and the immediate restoration of infrastructure.

Disaster mitigation reduces the impacts and risks of hazards. Disaster mitigation measures may be structural or nonstructural. The mitigation phase focuses on long-term measures for reducing the risk. The activities of rehabilitation phase include providing water supply, food safety, basic sanitation and personal hygiene to the victims of a disaster. 
Some of the major disasters around the world include:

- 2001 Gujarat earthquake.

- 2004 Indian Ocean tsunami.

- 2005 Hurricane Katrina.

- 2010 Haiti earthquake.

- 2011 Tohuku earthquake and tsunami.

- 2011 Newzealand earthquake.

Disaster management is a complex process involving local, national and international organizations. Disaster situations can be handled effectively by the co-ordinated efforts of these organizations. These organizations include The United Nations and its organizations, International committee of the Red Cross, International non-governmental agencies and other national organizations like National Disaster Management Authority (NDMA). NDMA has been set up for Disaster Management in India, with Prime Minister as its chairman. Disaster Management Authorities will be set up at the state and district levels to handle the disaster situation [4]. Disaster related problems can also be solved using operations research. OR is an applied science that utilizes mathematical techniques as tools to solve specific practical problems [11]. New advancements in the field of educational and research areas were introduced in US, to handle the disaster situation. This is known as Disaster Medical Education (DME). DME has received greater attention in the disaster management field. Three primary approaches employed in this field include standard classroom instruction, integration of basic game technology and the use of high fidelity live exercises [12].

\section{LITERARTURE REVIEW}

National Disaster Management Framework was developed in 2004. This framework can link various issues of structural and non-structural issues of prevention and preparedness, response and recovery and thereby provides effective risk mitigation and management [4]. Information management is an important phase of a disaster management system. Effective information management is a vital component of disaster response and relief. Accurate and timely information should be available before, during and after disasters. Here we discuss five major disaster management systems.

\section{EPIC [Empowering the Public with Information in Crisis]}

EPIC project promotes the use of social media channels for .mapping, visualizing and monitoring the hazard. The system is also known as Tweak-the-Tweet (TtT) [5]. Hash based syntax is used for efficient data extraction from twitter communications. TtT makes use of twitter platform for information gathering during emergencies, crisis and disasters. This system was developed in the year 2010. In this system the location sensor is exploited indirectly through the Twitter app. Twitters subscribe to the tweet broadcasts of other tweeters by 'following' them. Tweak-the-Tweet by Kate Starbird and project EPIC is licensed under a creative Commons Attribution 3.0 unported license [5].

\subsection{Crisis reporting Hashtag Syntax}

The users of the Tweak-the-Tweet system format their tweets with specific hashtags. These hashtags can be used to make information pieces that refer to \#location, \#status, \#damage and several other elements. This allows the computers to do a fixed round of processing on the information. The information obtained through hashtags is then processed to extract the location information, to create incident reports from tweets and to sort the reports into different types of categories. These processed tweets can be displayed on public web pages in varied formats. This allows users to view aggregate information about the disaster event.

\subsection{Example code of Tweak-the-Tweat}

Tweet-Before

Help needed in \#BethelFriday 12PM-7PM in the parking lot of Whitehill high school, Bethel \#VTresponse

Tweet-After

\#needhelp-Bethel \#time fri 12PM-7PM parking lot \#loc Whitehill high school, Bethel \#VTresponse

Tweak-the-Tweet system is designed as a role-based Publish/Subscribe system so that the co-workers can share information in case of emergencies. Twitter platform allows users to broadcast short, 140 character tweets to the listeners. Hash tags are used by twitters to tag or markup their tweets. Varieties of micro syntaxes have been proposed in order to increase the utility of the twitter platform. Microsyntax.org is a website established to record micro syntax suggestions. It also encourages discussions around different types of innovations.

\subsection{Advantages of Tweak-the-Tweet system \\ - Twitter communications have increased accessibility through mobile devices.}

- $\quad$ Tweak-the-Tweet system allows flexible delivery so that the publishers can choose the most appropriate communication technique for the urgent situation.

- Tweak-the-Tweet system provides role based subscription guidance.

Inspite of these advantages there exist a disadvantage for this system. There is an additional overhead of converting the normal messages to hash tags. Tweak-the-Tweet system allows the creation and support of adhoc communities of interest to address urgent situations. This system was used for a variety of weather emergency events like Haiti earthquake, Chile earthquake, Queensland flood etc which happened in the year 2010, 2011 [5].

\section{MRCCFR [Mobile Rich Media Communication Tool For First Responders]}

In emergency situations forces are fragmented and hence coordination is difficult from remote command centers. Normal communication networks are not applicable in case of emergencies. MRCCFR was intended for real time situational awareness by visual means [6]. This system was implemented as part of the INFRA (Innovative and Novel First Responders Applications Project). The system uses a Command and Control system often called a C2 system that merges and processes information sources from different sensors. This system visualizes information during an emergency situation and sends video annotation back and forth to first responders.A first responder is an employee who is part of an emergency service who usually arrives first at the affected area such as an accident, natural disaster or terrorist attack. Video annotation is the process of adding additional 
information like descriptive or self explanatory data to a video, related to its time and spatial dimensions. The Command Post application performs information integration and visualizes the data arriving from diverse information sources such as sensors, cameras and PDAs used in CI sites. The application collectively visualizes all information sent by First Responders (FRs).

The Command Post application is composed of two major subsystems. The first subsystem is the real time information merging visualization subsystem. This subsystem processes and manages real-time information sources and first responders at the critical site during an emergency situation. This subsystem performs selective visualization of the information according to the priority received or detected in data alerts and alarms. The second subsystem is the administrative one. This subsystem allows operators to input fundamental data in its database, author/edit rich media templates that are used for enhanced visual communication with FRs on site and retrieves past cases and history logs. Thus the C2 system serves as an enhanced location-based situational awareness and decision support tool. It also acts as a communication and alerting tool for the first responder teams. Fig 1 describes the various steps of the Command and control system.

Communication between various devices and information sources occurs through an adhoc wifi network. The C2 application is a distributed integrated emergency handling system. The application consists of a centralized information merging and communication hub and it runs on a web server with a number of sub components. These sub components work together to achieve information merging and visualization of the incoming information. The sub components provides location- based situational awareness and decision support and it also helps in bi-directional communication between the component post and the first responders on site. Thresholds and filtering are done to draw attention to data which is critical. In order to carry out communication between the Command and Control application and FRs, a set of alert icons and rich media templates are formally defined. Each alert icon corresponds to different emergency cases. Such alert icons and media templates, used in visual communications facilitate the immediate and correct understanding of the crisis situation.

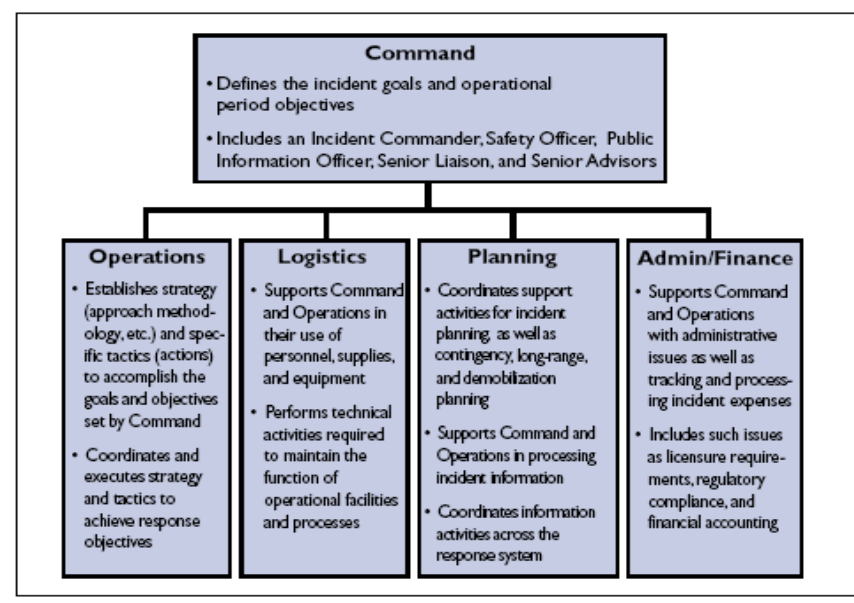

Fig 1: Overall System Architecture [6]

\subsection{C2 System Evaluation}

The system was evaluated at a number of occasions either via internal lab testing or in actual field trials. The complete $\mathrm{C} 2$ system has been evaluated qualitatively during a recent field trial. The trial was done at the M30 tunnel in Madrid, Spain in January 2011 [6]. The test sequence involved testing of both the real-time (annotated video receipt and alerts from FRs, FR vital sign information and alerts, FR locations real-time visualization, thermal imaging data receipt and alerts) and administrative (user/CI data management, history log subsystems, template administration, etc.). Users were stationed in the M30 emergency tunnel as well as in the Command Center during tests. The developed C2 system was deployed and tested successfully - the sending of a $721 \mathrm{~KB}$ annotated video from an FR to the Command post took 11 seconds. The access points are positioned within a $1 \mathrm{~km}$ distance. The main limitation is that the system rated only 60 percent accuracy.

\section{6. iGaDs (Intelligent Guards Against Disasters)}

iGaDs are devices that can receive and process alert messages. The term iGaDs refers to embedded devices, systems and applications. These devices can authenticate and process standard disaster warning messages and can respond by taking appropriate actions. iGaDs helps to enhance the preparedness for disasters. iGaDs can be used in a variety of disaster situations. An $\mathrm{iGaD}$ in a smart home can shut down the natural gas intake valve to prevent fire disaster [7]. Such an $\mathrm{iGaD}$ can be configured to respond to strong tornado warnings as well as earthquake warnings. Software iGaDs are CAPaware applications that run on computers, smart phones and other platforms with sufficient computing power and memory space.

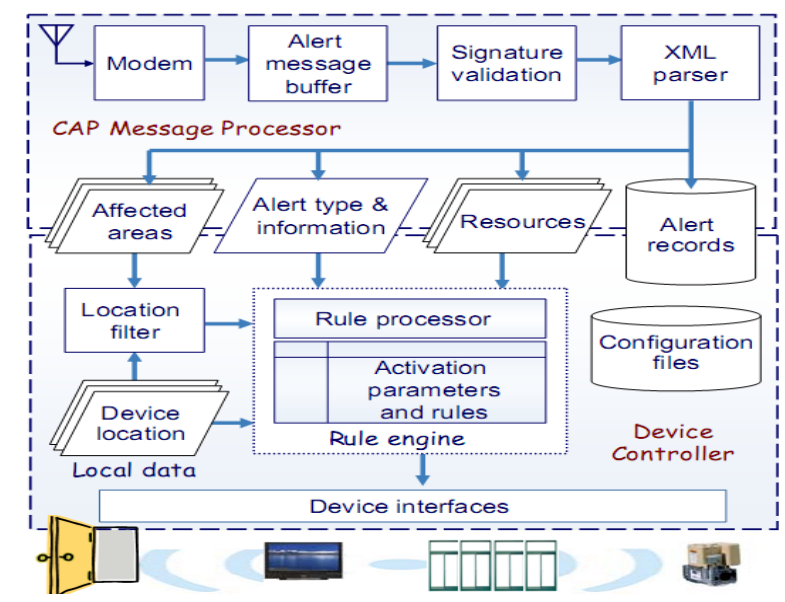

Fig 2: General Structure of iGaDs System [7].

Fig 2 shows the general structure of iGaDs system. A smart device is attached to the building. The device intelligently interprets the signals and gives disaster alerts to a group of people. To give different response to the same alert messages, iGaDs of the same type are to be used. Thus iGaDs devices must be highly configurable and customizable. iGaDs are created for natural disaster prediction and detection. The use of iGaDs enables generation of machine-readable early disaster alerts over all communication channels. The system can be implemented by attaching a smart device to the building. Each $\mathrm{iGaD}$ needs to evaluate rules governing whether it should respond to the alert extracted from the current message. Rather than using existing rule engines, the current version of $\mathrm{iGaD}$ prototype uses a lightweight scheme to processing the rules [6]. iGaDs based on this scheme can be easily customized. An alert message may include additional parameters of the event such as expiration times, image, text 
descriptions and so on. Some iGaDs (e.g., those that provide emergency alert functions of building management systems) can use them as suggested by the CAP-EAS implementation guidelines [7] in the generation of their responses. The main disadvantage of iGaDs system is that it is limited to a particular building.

\section{UBALERT DISASTER APPLICATION}

UbAlert is a global social network that is intended to save lives by sharing the knowledge about the disaster situation. UbAlert app provides all the necessary information that is required regarding the disaster event. The data is collected from verified sources to get access to everything about the disaster event. Alerts contain basic event details like impact statistics, maps, images, videos and more. The alert reports can be shared through email, facebook and twitter to bring the victims out of the hazard.

The main goal of this app is to create a disaster alerting network. This app makes use of the data that is combined from global institutions and data providers. It is an emergency warning platform that validates the reliability of the reported content. UbAlert gives power to the people. The UbAlert checks to see who all are within the impact area and is interested in the alert. The information is then sent to those people who are acts as first responders. The basic features of UbALert include:

- It consists of an interactive map.

- The application sorts events by severity/popularity/credibility.

- Reports an event by submitting a photo or video.

- Share events by email or through other social media channels.

- The alerts are saved to the "watch list" for quick viewing.

- $\quad$ Adjust text size.

- To prevent fraudulent posts, the application provides a feedback regarding credibility of the post.

The settings of the application can be adjusted in such a way that it can adjust display distance in kilometers or meters or heads.

The UbAlert application provides the most up-to-date and accurate incident alert notification to save lives of the victims in the disaster situation [8]. UbAlert provides timely notification of natural human caused disaster events. UbAlert is not a news agency and doesn't normally originate stories by itself. UbAlert collects disaster and hazard information from various sources that are accurate and reliable. These include various governmental or international agencies, university programs or third party information integrators. The UbAlert application integrates the data from various organizations and provides a single window for global disaster alerting and notification.There are certain limitations for UbAlert disaster application. The use of social media sites at the time of emergency will consume a lot of time. Due to this the rescue operation may ge t delayed [10].

\section{BRIDGE [Bridging Resources and Agencies In Large Scale Emergency Management]}

The BRIDGE project is an ongoing project. The main goal of BRIDGE is to increase the safety of citizens by developing technical and organizational solutions that significantly improves emergency management. This ensures interoperability, harmonization and co-operation among stakeholders on the technical and organizational level. It delivers an ad-hoc network infrastructure. The system focuses on the requirements evolving from emergency scenarios. The data sources, networks and systems are integrated and are defined under BRIDGE middleware. The content management system provided by BRIDGE fosters interoperability of data, providing meaningful and reliable information [9].

BRIDGE is committed to an iterative user-centered approach incorporating the domain requests. BRIDGE project was started in APRIL 2011. The project is planned for duration of 4 years [9]. The system builds a bridge between multiple first responder organizations. The system is expected to be a part of large scale emergency management organizations for contributing to effective relief efforts. The system provides interaction between developers and non-technical people through 'Concept Cases (CCs)'. A Concept Case (CC) is a specific tool that meets certain needs of some participants in crisis response operations. The use of $\mathrm{CCs}$ can align the results of the BRIDGE project within the users' requirements and tasks. The Concept Cases of BRIDGE project is known as BRIDGE SWARM concept case. The SWARM Concept Case integrates the Master Table with a general purpose smartphone application through a Publish/subscribe service provided by the BRIDGE middleware [13].

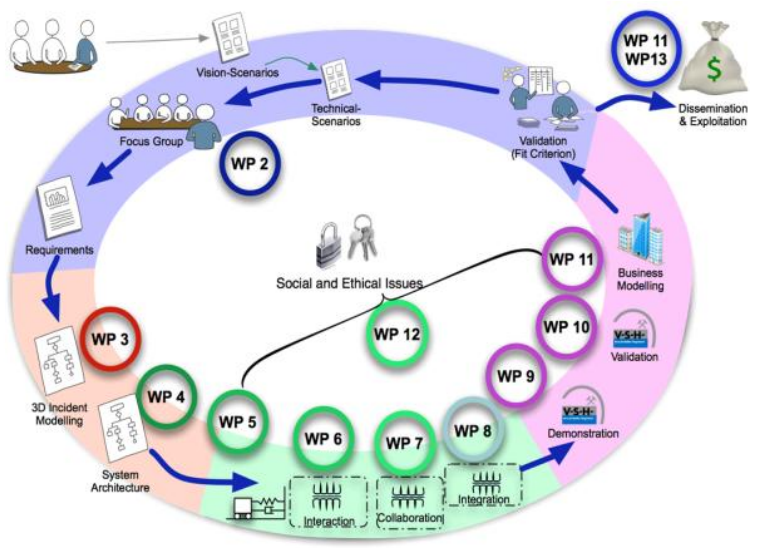

Fig 3: BRIDGE Work Pages [9].

The middleware of the BRIDGE project is service oriented. It offers services like: communication, data/model management, security/trust and orchestration. Some services are already demonstrated. These include: messaging, network management, workflow management, publish/subscribe, triggering and eventing, service catalogue, identification, shared data store. Further work associated with BRIDGE explores ethical, legal and social consequences of new practices of public engagement in emergency response via social media. In order to accomplish its goals, BRIDGE has been divided into 13 different work pages, as shown in Fig 3 . 


\section{CONCLUSION}

Communication during disaster time is very crucial for both rescue team and victim. Emergency never comes with prior intimation. The disaster management system is intended to function in case of emergencies in the society. The emergencies include fire, medical emergencies, accident and external emergencies like earthquake, floods, storm etc. In this paper a survey on the various disaster management systems has been performed. Firstly, the need of developing a disaster management system is discussed. A comprehensive evaluation of the existing disaster management system is also discussed. In all the above discussed disaster management systems, the application of each system is restricted to a particular area or a building. In T2T system and the UbAlert disaster application need the assistance of social media to post photos and share information about the disaster situation. Thus as a future scope an efficient disaster management system is to be developed which can be used easily by the disaster management teams and the victims trapped in the disaster situation. A mobile application can be developed which can be make use of the GPS in phone to track the location of the disaster area.

\section{REFERENCES}

[1] Perera, I., et al., Managing 3D Multi User Learning Environments - A Case Study on Training Disaster Management. International Journal of Emerging Technologies in Learning (iJET), 2012. 7(3): p. 25-34.

[2] Yadav, S. 4000 youths to get disaster management training. 2013 [cited2013 September]; Available from http://articles.timesofindia.indiatimes.com/20130825/gur gaon/41445127_1_disaster-management-uttarakhandfloodsyouth

[3] Hyogo framework for Action 2005-2015 : Building the Resilence of nations and communities to disasters [online] Available: http://www.preventionweb.net/english/professional/publi cations/v.php?id=1037\&pid:22\&pih:2.

[4] India Disaster Knowledge Network [online] Available: http://www.saarcsadkn.org/countries/india/disaster_profile.aspx.

[5] K. Starbird and J. Stamberger, "Tweak the Tweet: Leveraging Microblogging Proliferation with a Prescriptive Syntax to Support Citizen Reporting," in the 7th International ISCRAM Conference, Seattle, USA, 2010 ..

[6] M.Bakopoulos, S. Tsekeridou, E. Giannaka, Z.-H. Tan, and R. Prasad,"Command \& Control: Information Merging, Selective Visualization and Decision Support for Emergency Handling," in ISCRAM, Lisbon, Portugal, 2011.

[7] Y.Z.Ou, C.M.Huang, C.T. Hu, J.W.S. Liu, E.T.H. Chu, C.S. Shih, "An Asynchronous Message Delivery Service for iGaDs", in Proceedings of International Workshop on Extending Seamlessly to the Internet of Things, July 2013.

[8] Terms and conditions of use of ubAlert disaster network [online] Available: www.ubalert.com

[9] SINTEF Published Article on BRIDGE in Canadian GazetteMagazine[online]Available:http://www.rcmp.gc. ca/gazette/vol76no4/cover-dossier/norway-norvege eng.htm.

[10] Rehka Jadhav, Jwalant Patel, Darshan Jain, Suyash Phadhtare, "Emergency Management System Using Android Application ," International Journal of Computer Science and Information Technologies, Vol. 5(3), 2014,.

[11] Yiping Jiang, Yufei Yuan, Kai Huang, Lindu Zhao, "Logistics for Large-scale Disaster Response: Achievements and challeneges", $201245^{\text {th }}$ Hawaii International Conference on System Sciences.

[12] AJ Hayes, Jessica Lancaster, Zeno Franco, Anne Kissack, "Disaster Medical Education and Simulated Crisis Events: A translational approach", Proceedings of the $9^{\text {th }}$ International ISCRAM Conference-Vancouver, Canada, April 2012.

[13] BRIDGE SWARM Concept Case [online] Available: http://www.bridgeproject.eu/content/bridge_swarm_flyer .pdf.

[14] Indika Perera, Dulani Meedeniya, Indushree Banerjee, Joydeep Choudhury, "Educating Users for Disaster Management", 2013 IEEE International Conference in MOOC, Innovation and Technology in Education (MITE). 